\title{
MULTIDRUG-RESISTANT TUBERCULOSIS IN PATIENTS WITHOUT HIV INFECTION
}

\author{
Edward E. Telzak, M.D., Kent Sepkowitz, M.D., Peter Alpert, M.D., Sharon Mannheimer, M.D., \\ Franz Medard, M.D., WafaA El-Sadr, M.D., Steve Blum, Ph.D., A. Gagliardi, M.D., \\ Nadim Salomon, M.D., and Glenn Turett, M.D.
}

\begin{abstract}
Background. Investigations of outbreaks of multidrug-resistant tuberculosis have found low rates of treatment response and very high mortality, and they have mainly involved patients with advanced human immunodeficiency virus (HIV) infection. For patients without HIV infection, one study reported an overall rate of response to treatment of 56 percent, and the mortality from tuberculosis was 22 percent. We investigated treatment response and mortality rates in 26 HIV-negative patients in New York with multidrug-resistant tuberculosis.

Methods. We obtained detailed data from seven teaching hospitals in New York City on patients with multidrug-resistant tuberculosis - defined as tuberculosis resistant at least to isoniazid and rifampin - who were HIV-negative on serologic testing. Lengths of times from diagnosis to the initiation of appropriate therapy and from the initiation of appropriate therapy to conversion to negative cultures were assessed. Therapeutic responses were evaluated by both microbiologic and clinical criteria.
\end{abstract}

$\mathrm{T}$ HE emergence of multidrug-resistant tuberculosis has been well documented in both New York City and the rest of the United States. ${ }^{1-3}$ Though New York City had the highest rate in a national survey of reported tuberculosis cases in the first quarter of 1991, cases of multidrug-resistant tuberculosis were reported from 13 states and 35 of the nation's counties (1.1 percent). ${ }^{3}$

Previous studies have found that patients with drugresistant tuberculosis have lower response rates than patients with drug-susceptible isolates. ${ }^{4,5}$ Recent investigations of outbreaks of multidrug-resistant tuberculosis have found an extraordinarily high case fatality rate, with the median mortality being reached between 4 and 16 weeks. $^{6-9}$ In almost all instances, these outbreaks have involved patients severely immunosuppressed by infection with the human immunodeficiency virus (HIV). More recent data suggest that outcomes can be improved if patients promptly start to receive two or more drugs that have in vitro activity against the multidrug-resistant isolates. ${ }^{10-12}$

The expected outcome in patients not infected with HIV who have multidrug-resistant tuberculosis is less well defined. However, a widely cited study of $171 \mathrm{HIV}-$ negative patients treated from 1973 to 1983 at the National Jewish Center for Immunology and Respiratory Medicine found an overall response rate of 56 percent and a mortality rate attributable to tuberculosis of 22 percent. ${ }^{13}$

In New York City, because of the recent dramatic in-

From the Bronx-Lebanon Hospital Center, Bronx, N.Y. (E.E.T., S.B., G.T.); St. Clare's Hospital and Health Center, New York (K.S.); New York Hospital-Cornell Medical Center, New York (K.S., S.M.); Harlem Hospital Center, New York (F.M., W.E.-S.); Montefiore Medical Center and North Central Bronx Hospital, Bronx, N.Y. (P.A.); St. Vincent's Medical Center, New York (A.G.); and Beth Israel Medical Center, New York (N.S.). Address reprint requests to Dr. Telzak at the Division of Infectious Diseases, Bronx-Lebanon Hospital Center, Albert Einstein College of Medicine, 1650 Grand Concourse, Bronx, NY 10457.
Results. Between March 1991 and September 1994, 26 HIV-negative patients were identified and treated. Of the 25 patients for whom adequate data were available for analysis, 24 (96 percent) had clinical responses; all 17 patients for whom data on microbiologic response were available had such a response. The median times from diagnosis to the initiation of appropriate therapy and from the initiation of therapy to culture conversion were 44 days (range, 0 to 181) and 69 days (range, 2 to 705 ), respectively. Side effects requiring the discontinuation of medication occurred in 4 of 23 patients (17 percent) who were treated with second-line antituberculosis medications. The median follow-up for the 23 patients who responded and who received appropriate therapy was 91 weeks (range, 41 to 225).

Conclusions. In this report from New York City, HIVnegative patients with multidrug-resistant tuberculosis, contrary to previous reports, responded well to appropriate chemotherapy, both clinically and microbiologically. (N Engl J Med 1995;333:907-11.)

crease in cases of tuberculosis and multidrug-resistant tuberculosis, we have had the opportunity to evaluate patients with multidrug-resistant tuberculosis who are not HIV-infected. We describe our experience with 25 patients whose clinical characteristics and outcomes differed dramatically from those of previously published studies.

\section{Methods}

We identified cases of multidrug-resistant tuberculosis — defined as tuberculosis caused by Mycobacterium tuberculosis resistant at least to isoniazid and rifampin - through a network of hospital-based physicians who treat patients with tuberculosis at seven teaching hospitals in New York City, including five private hospitals (Bronx-Lebanon Hospital Center, Montefiore Medical Center, New York Hospital, St. Clare's Hospital, and Beth Israel Medical Center) and two public ones (Harlem Hospital Center and North Central Bronx Hospital). Only patients who were found to be HIV-negative by routine serologic testing were included in the review. For patients who met these criteria, detailed data-abstraction forms were completed by physicians at the hospitals where the patients initially received the diagnosis of multidrug-resistant tuberculosis. The forms were designed to obtain information on demographic characteristics, risk factors for tuberculosis infection, the extent and verification of tuberculosis, and the response to, and toxicity of, antituberculosis therapy.

The date of diagnosis of multidrug-resistant tuberculosis was defined as the date of collection of the first specimen from which multidrug-resistant tuberculosis was isolated. The time to the initiation of appropriate therapy was defined as the time from diagnosis to the institution of a regimen containing at least two drugs with in vitro activity against the isolate. For one patient, appropriate therapy was begun 63 days before the date of diagnosis. In the analysis, this patient was assigned a value of zero days from diagnosis to appropriate treatment. Another patient was in remission more than three years after his initial diagnosis, without ever having received appropriate treatment. He was removed from this analysis. The time to culture conversion was defined as the time from the start of appropriate therapy to the collection of the first in a series of two or more consecutive negative cultures at least two weeks apart. A therapeutic response was evaluated on the basis of both microbiologic and clinical criteria. A microbiologic response was defined as negative results of at least two consecutive sputum cultures at least two weeks apart. A clinical re- 
sponse was defined as the resolution of signs and symptoms associated with the diagnosis of tuberculosis. Patients were considered to have completed therapy if they had received a total of at least 18 months of appropriate therapy or if they had received at least 12 months of appropriate therapy after the conversion of their sputum cultures to negative; 13 patients satisfied this criterion. Three additional patients categorized as having completed therapy discontinued appropriate treatment against medical advice after they had clinical or bacteriologic responses or both. The duration of follow-up was defined as the interval between the date appropriate therapy was initiated and the date of the last known contact.

\section{Microbiologic Methods}

Standard procedures were used according to the practice of each hospital. Five hospitals used their own laboratories to identify $M$. tuberculosis and to test for susceptibility to first-line antituberculosis medications, one hospital sent specimens to a commercial laboratory, and one hospital sent specimens to the laboratories of the New York City Department of Health. Bactec radiometric mediums (Becton Dickinson, Towson, Md.) were used by five of the laboratories, and solid mediums were used by the other two. ${ }^{14}$ Tests for susceptibility to pyrazinamide were not routinely performed until April 1992, and results are included here when they were available. Testing for susceptibility to second-line antituberculosis medications and retesting for susceptibility to first-line drugs were performed for 20 patients $(80$ percent) by a referral laboratory at one of four institutions: the Centers for Disease Control and Prevention, the New York City Department of Health, the National Jewish Center for Immunology and Respiratory Medicine, and the West Haven Veterans Affairs Hospital. ${ }^{14}$ For specimens tested by referral laboratories, multidrug resistance was defined by the growth on drug-containing mediums $(0.2 \mu \mathrm{g}$ of isoniazid per milliliter and $1.0 \mu \mathrm{g}$ of rifampin per milliliter) of more than 1 percent of the colonies that grew on a drug-free (control) medium.

\section{RESULTS}

Between March 1991 and September 1994, more than 200 patients received diagnoses of multidrugresistant tuberculosis and were treated at the seven hospitals. The overwhelming majority of these patients were infected with HIV. During this interval, 26 HIVnegative patients with multidrug-resistant tuberculosis were identified and treated; for 25 of these adequate data were available for analysis. Case finding was complete at all seven hospitals. One hospital cared for seven patients, one for six, one for four, one for three, two for two, and one for one.

The demographic characteristics of the 25 patients are shown in Table 1. The median age at the time of diagnosis was 37 years, and 14 patients (56 percent) were male. Fifteen patients (60 percent) were black, four (16 percent) were Hispanic, four (16 percent) were Asian, and two (8 percent) were white. Of the 10 patients born outside the United States or Puerto Rico, 5 were born in Asia, 3 in Latin America, 1 in Africa, and 1 in Europe.

Previously established risk factors for multidrugresistant tuberculosis were identified in 17 of the patients (68 percent). Eight patients had a history of inadequately treated tuberculosis, and nine had known exposures to patients with multidrug-resistant tuberculosis. Of these nine, eight were health care workers and one was a patient hospitalized during a nosocomial outbreak of multidrug-resistant tuberculosis. Among the health care workers, five were physicians, two were nurses, and one was a medical administrator. An addi-

\begin{tabular}{lc}
$\begin{array}{c}\text { Table 1. Demographic Characteristics of } 25 \\
\text { HIV-Negative Patients with Multidrug- } \\
\text { Resistant Tuberculosis. }\end{array}$ \\
\hline \hline \multicolumn{2}{c}{ No. (\%) } \\
CHARACTERISTIC \\
Age (yr)* \\
$<25$ \\
$25-44$ \\
$\geqslant 45$ \\
Sex \\
Male & $2(8)$ \\
Female & $8(60)$ \\
Race or ethnic group & $14(32)$ \\
Black & $11(44)$ \\
Hispanic & $15(60)$ \\
Asian & $4(16)$ \\
White & $4(16)$ \\
& $2(8)$
\end{tabular}

tional three patients were residents of the New York City shelter system for the homeless.

All but four patients (84 percent) had disease localized to the lung or pleura; one had both pulmonary and peritoneal disease, and three had extrapulmonary disease alone (in the lymph nodes, the lumbar vertebrae, and the soft tissues of the scrotum). Nineteen of the 25 patients had no underlying disease before the diagnosis of tuberculosis; of the remaining 6,2 had diabetes mellitus and end-stage renal disease, 2 had diabetes alone, and 1 patient each had severe rheumatoid arthritis and adenocarcinoma of the lung.

Twenty-four patients (96 percent) had clinical responses. One woman with diabetes mellitus and endstage renal disease died five days after standard doses of isoniazid, rifampin, ethambutol, and pyrazinamide were initiated. Results of susceptibility tests of her isolate became available post mortem and indicated resistance to isoniazid, rifampin, streptomycin, and ethambutol. No autopsy was performed, but the clinical impression was that she died of overwhelming tuberculosis.

The patterns of susceptibility to the five first-line antituberculosis medications and the treatment regimens used for the 23 patients with responses who received appropriate therapy are summarized in Table 2 . Twelve of the isolates (52 percent) were resistant to four or five of the first-line antituberculosis agents. Eight patients had isolates that were resistant to isoniazid and rifampin only. Susceptibility testing was not done for pyrazinamide in eight patients.

Twenty-three patients who responded were ultimately treated with at least three drugs that had in vitro activity against their isolates, and all received prolonged treatment with a quinolone. Radiographic abnormalities and clinical symptoms resolved without treatment in the remaining patient with a response.

Three patients underwent therapeutic lobectomies at the discretion of the primary treating physicians. Cultures for one patient became negative only after a lobectomy, despite approximately 15 months of appropriate chemotherapy. The other two patients had culture-negative sputum specimens before surgery, but 
Table 2. Profile of Susceptibility to First-Line Antituberculosis Drugs and Treatment Regimens for 23 Patients with Multidrug-Resistant Tuberculosis Who Responded to Drug Treatment and Who Received Appropriate Therapy.

\begin{tabular}{|c|c|c|c|c|c|c|c|c|c|c|}
\hline \multirow[t]{2}{*}{ FIRST-Line DRUGS } & $\begin{array}{c}\text { No. of } \\
\text { PatiEnTS } \\
\text { Resistant }\end{array}$ & & & & SECONI & -LiNE DRUGS & & & & \\
\hline & & ETHAMBUTOL & PYRAZINAMIDE & AMINOGLYCOSIDE* & CAPREOMYCIN & QUINOLONE $\dagger$ & CYCLOSERINE & ETHIONAMIDE & PAS & CLOFAZIMINE \\
\hline & & \multicolumn{9}{|c|}{ number of patients } \\
\hline Isoniazid and rifampin & $8 \S$ & 8 & 8 & 6 & 0 & 8 & 0 & 3 & 0 & 0 \\
\hline $\begin{array}{l}\text { Isoniazid, rifampin, and } \\
\text { streptomycin }\end{array}$ & 1 & 1 & 1 & 0 & 1 & 1 & 0 & 1 & 1 & 0 \\
\hline $\begin{array}{l}\text { Isoniazid, rifampin, and } \\
\text { ethambutol }\end{array}$ & 1 & 0 & 1 & 0 & 1 & 1 & 1 & 1 & 0 & 0 \\
\hline $\begin{array}{l}\text { Isoniazid, rifampin, and } \\
\text { pyrazinamide }\end{array}$ & 1 & 1 & 0 & 1 & 0 & 1 & 0 & 0 & 0 & 0 \\
\hline $\begin{array}{l}\text { Isoniazid, rifampin, pyrazina- } \\
\text { mide, and ethambutol }\end{array}$ & $2 \mathbb{I}$ & 0 & 0 & 1 & 1 & 2 & 1 & 2 & 1 & 1 \\
\hline $\begin{array}{l}\text { Isoniazid, rifampin, strepto- } \\
\text { mycin, and ethambutol }\end{array}$ & $3 \|$ & 0 & 2 & 2 & 0 & 3 & 2 & 1 & 1 & 0 \\
\hline $\begin{array}{l}\text { Isoniazid, rifampin, pyrazina- } \\
\text { mide, and streptomycin }\end{array}$ & 1 & 1 & 0 & 0 & 1 & 1 & 1 & 0 & 0 & 0 \\
\hline $\begin{array}{l}\text { Isoniazid, rifampin, strepto- } \\
\text { mycin, ethambutol, and } \\
\text { pyrazinamide }\end{array}$ & $6^{* *}$ & 0 & 0 & 1 & 4 & 6 & 6 & 5 & 1 & 1 \\
\hline
\end{tabular}

*The aminoglycosides used were streptomycin, kanamycin, and amikacin. For patients given more than one parenteral agent (aminoglycoside and capreomycin), the one given for the longer course is indicated.

$\dagger$ The quinolones used were ciprofloxacin and ofloxacin.

$\doteqdot$ PAS denotes $p$-aminosalicylic acid.

$\S$ Two patients received five drugs; five patients, four drugs; and one patient, three drugs.

||Two patients received four drugs; and one patient, three drugs.

TIOne patient received three drugs.

surgery was performed because of localized cavitary disease found on chest radiography.

The median time from the diagnosis of multidrugresistant tuberculosis to the initiation of appropriate therapy was 44 days (range, 0 to 181) for the 23 patients who ultimately received at least three drugs with in vitro activity against the multidrug-resistant isolate. All 17 patients for whom data on microbiologic response were available had such responses. The median time to a microbiologic response was 69 days (range, 2 to 705), with a mean of 126 days. The mean was influenced by two extreme values of 705 and 496 days. The first value was that of a patient who was noncompliant for more than 2 years but who subsequently responded after 90 days of appropriate therapy that was directly observed. The patient with the second value responded only after a lobectomy, despite appropriate and prolonged chemotherapy. All 17 patients who had microbiologic responses also had clinical responses, with the resolution of signs and symptoms.

Of the eight patients for whom microbiologic response could not be determined, four did not have follow-up specimens obtained because of the site of disease (lymph nodes, vertebrae, soft tissue of the scrotum, or pleura), and one patient died before follow-up specimens could be obtained. Follow-up sputum specimens were not obtained from three patients. Seven of these eight patients had clinical responses.

As of March 31, 1995, 16 patients (64 percent) had completed a course of therapy without evidence of relapse (Table 3). Seven patients (28 percent) were in remission while still receiving therapy, and one remained in remission without having ever received appropriate therapy. The 16 patients who had completed therapy

received quinolones for a median of 568 days (range, 150 to 787). In addition, 13 of these 16 patients received parenteral therapy with either an aminoglycoside or capreomycin (median, 252 days; range, 34 to 595); 8 received ethionamide (median, 570 days; range, 119 to 766 ); and 7 received cycloserine (median, 559 days; range, 119 to 595).

The median duration of follow-up from the time that appropriate therapy was instituted to the date of last contact for the 23 patients with responses who received treatment was 91 weeks (range, 41 to 225). For the 17 patients who had microbiologic responses, the median duration of follow-up from the time of microbiologic response was 81 weeks (range, 30 to 146). For the 16 patients who completed therapy, the median duration of follow-up after the initiation of appropriate therapy was 121 weeks, and after the completion of therapy, 22 weeks (range, 0 to 52 ).

Eight patients had a history of tuberculosis; seven of them completed therapy during the study period. These patients had had tuberculosis for a median of 2.5 years

Table 3. Outcomes of 25 Patients with MultidrugResistant Tuberculosis.

\begin{tabular}{|c|c|c|}
\hline OUTCOME & No. $(\%)$ & $\begin{array}{l}\text { MEDIAN } \\
\text { FOLLOW-UP } \\
\quad \text { (WK })\end{array}$ \\
\hline $\begin{array}{l}\text { Completed a course of therapy and was } \\
\text { without evidence of disease }\end{array}$ & $16(64)$ & 121 \\
\hline Was under treatment and in remission & $7(28)$ & 62 \\
\hline $\begin{array}{l}\text { Was in remission without appropriate } \\
\text { treatment }\end{array}$ & $1(4)$ & 199 \\
\hline Died & $1(4)$ & NA* \\
\hline
\end{tabular}

*NA denotes not applicable. This patient died five days after admission without receiving appropriate therapy. 
(range, $<1$ to 6 ) and had received a median of 3.5 antituberculosis drugs before the initiation of appropriate therapy for their multidrug-resistant tuberculosis. The median time to a microbiologic response in the $7 \mathrm{pa}-$ tients for whom data were available was 79 days, as compared with 62 days in the 10 patients with available data and no history of tuberculosis.

Serious side effects requiring the discontinuation of second-line antituberculosis medication occurred in four patients (17 percent) (Table 4). Nineteen patients had no serious adverse reactions. In addition, 13 patients had six or more months of parenteral therapy with an aminoglycoside or capreomycin or both. Ototoxicity in one patient necessitated the discontinuation of kanamycin.

\section{Discussion}

Drug-resistant and multidrug-resistant tuberculosis is a growing problem in the United States. The emergence of resistance may be due to either patient noncompliance ${ }^{1,15}$ or the transmission of already resistant strains. ${ }^{16-18}$ The Centers for Disease Control and Prevention conducted a nationwide susceptibility survey of tuberculosis cases reported in 1991 and found that 14.2 percent were resistant to at least one drug and 3.5 percent were resistant to both isoniazid and rifampin and therefore considered to be multidrug-resistant. ${ }^{3}$

The optimal therapy for drug-susceptible tuberculosis is well established after numerous trials comprising thousands of patients. The optimal therapy for drugresistant tuberculosis, particularly multidrug-resistant tuberculosis, is less well studied and standardized. Mitchison and Nunn reviewed the outcomes of patients with drug-resistant tuberculosis in 12 controlled trials conducted in Africa, Hong Kong, and Singapore. ${ }^{4}$ Of 8212 patients studied, 1041 (12.7 percent) had tuberculosis with initial resistance to at least one drug and 256 (3.1 percent) had tuberculosis resistant to both isoniazid and streptomycin. Only 11 isolates were resistant to rifampin. Chemotherapy failed in 5 of the 11 patients

Table 4. Side Effects of Second-Line Antituberculosis Medications Leading to the Withdrawal of Treatment in 4 of 23 Patients Treated for Multidrug-Resistant Tuberculosis.*

\begin{tabular}{lcc}
\hline \hline Drug & $\begin{array}{c}\text { No. with Reaction/ } \\
\text { No. Given Drug }\end{array}$ & Reaction \\
Amikacin & $0 / 7$ & - \\
Capreomycin & $0 / 8$ & - \\
Kanamycin & $1 / 2$ & Ototoxicity \\
Cycloserine & $1 / 11$ & Seizure \\
Ethionamide & $1 / 13$ & Vomiting \\
Ciprofloxacin & $0 / 13$ & - \\
Ofloxacin & $0 / 10$ & - \\
$p$-Aminosalicylic acid & $1 / 4$ & Nausea \\
Clofazimine & $0 / 2$ & - \\
\hline
\end{tabular}

*Nineteen of the 23 patients ( 83 percent) had no toxic effects, and 13 patients received $\geqslant 6$ months of parenteral aminoglycosides or capreomycin or both. with tuberculosis resistant to rifampin, and 3 of the remaining 6 patients relapsed after therapy was stopped. These responses are in contrast to the high response rates and few relapses that were seen in patients whose disease was initially resistant to drugs other than rifampin. The authors attributed these findings to the excellent sterilizing activity of both rifampin and pyrazinamide and to the ability of rifampin to help prevent the emergence of drug resistance during treatment.

A recently published study described the outcomes of 171 patients with multidrug-resistant tuberculosis treated over an 11-year period from 1973 to $1983 .{ }^{13}$ These patients had tuberculosis for a median of six years and had received a median of six drugs before being referred to the National Jewish Center for Immunology and Respiratory Medicine in Denver. Patients were shedding tubercle bacilli that were resistant to a median of six drugs. Outcomes in this group were poor: of 134 patients for whom adequate follow-up data were available, 87 (65 percent) initially responded to chemotherapy and 47 (35 percent) had no response, as manifested by persistently positive cultures. The overall response rate, including relapses, was 56 percent, and the mortality attributable to tuberculosis was 22 percent.

In studies of outbreaks in the early 1990s, patients with the acquired immunodeficiency syndrome (AIDS) and multidrug-resistant tuberculosis had a median survival of 4 to 16 weeks. ${ }^{6,7}$ In one report, those who were HIV-infected but without a diagnosis of AIDS had a median survival of 14 months. ${ }^{9}$ Recent reports have described improved outcomes when HIV-infected patients received prompt diagnoses and were treated rapidly with appropriate medications..$^{10-12}$ In our series, the median time from the diagnosis of multidrug-resistant tuberculosis to the initiation of appropriate therapy was 44 days. This relatively short period, as compared with those reported previously, is probably due to three factors: the practice of starting treatment with isoniazid, rifampin, pyrazinamide, and ethambutol for all patients with tuberculosis before the results of susceptibility testing were available; the use of Bactec radiometric mediums; and maintenance of a high index of suspicion for drug-resistant tuberculosis.

We have shown that HIV-negative patients with multidrug-resistant tuberculosis can be expected to have a good response to medical therapy. All of our patients who received appropriate therapy have had clinical and microbiologic responses. There were no relapses or treatment failures during a median follow-up period of 91 weeks. It is still possible that some of these patients will relapse. This group, however, in addition to having the benefits of quinolone therapy, probably represents patients of a different type from those described in the Denver study. The Denver patients, as we have mentioned, were heavily pretreated and were referred after treatment by physicians in their own communities had failed. Many of our patients, on the other hand, probably had primary disease; in fact, only eight of the patients (32 percent) had a history of tuberculosis. 
As recent studies based on restriction-fragment-length polymorphisms have demonstrated, recent transmission accounts for as much as 40 percent of cases of adult tuberculosis in certain urban populations. ${ }^{16,17} \mathrm{It}$ may be that our results reflect the advantage of treating primary resistant disease, rather than disease in persons exposed intermittently to antituberculosis medication for years, resulting in secondary resistance and damaged lungs. Even our patients with previous tuberculosis, all of whom responded, represented a group different from those treated in the Denver series. They had tuberculosis for a shorter period (2.5 years, vs. 6 for the Denver patients) and were not as heavily pretreated (median number of drugs, 3.5, vs. 6 in Denver). Lastly, as we saw in one of our patients, primary tuberculosis, even when due to a multidrug-resistant strain, may resolve with no effective chemotherapy, although the risk of reactivation clearly remains.

Since this retrospective review is based on the experience of seven hospitals, laboratory techniques and treatment regimens varied. Nevertheless, we conclude that contrary to previous reports, patients without HIV infection who contract multidrug-resistant tuberculosis can be expected to respond to appropriate chemotherapy, both clinically and microbiologically. As in other studies, serious drug toxicity is not unusual, and safer agents are needed. Assessment of the long-term efficacy of this approach awaits several more years of patient follow-up.

We are indebted to Drs. Jonathan W.M. Gold and Barkat A. Fazal for their critical comments, and to Olga Zerafa for assistance in the preparation of the manuscript.

\section{REFERENCES}

1. Frieden TR, Sterling T, Pablos-Mendez A, Kilburn JO, Cauthen GM, Dooley SW. The emergence of drug-resistant tuberculosis in New York City. N Engl J Med 1993;328:521-6.

2. Sepkowitz KA, Telzak EE, Recalde S, Armstrong D, New York City Area Tuberculosis Working Group. Trends in the susceptibility of tuberculosis in New York City, 1987-1991. Clin Infect Dis 1994;18:755-9.
3. Bloch AB, Cauthen GM, Onorato IM, et al. Nationwide survey of drugresistant tuberculosis in the United States. JAMA 1994;271:665-71.

4. Mitchison DA, Nunn AJ. Influence of initial drug resistance on the response to short-course chemotherapy of pulmonary tuberculosis. Am Rev Respir Dis 1986; 133:423-30.

5. Vall-Spinosa A, Lester W, Moulding T, Davidson PT, McClatchy JK. Rifampin in the treatment of drug-resistant Mycobacterium tuberculosis infections. N Engl J Med 1970;283:616-21.

6. Nosocomial transmission of multidrug-resistant tuberculosis among HIVinfected persons - Florida and New York, 1988-1991. MMWR Morb Mortal Wkly Rep 1991;40:585-91.

7. Outbreak of multidrug-resistant tuberculosis at a hospital - New York City, 1991. MMWR Morb Mortal Wkly Rep 1993;42:427-34.

8. Edlin BR, Tokars JI, Grieco MH, et al. An outbreak of multidrug-resistant tuberculosis among hospitalized patients with the acquired immunodeficiency syndrome. N Engl J Med 1992;326:1514-21.

9. Fischl MA, Daikos GL, Uttamchandani RB, et al. Clinical presentation and outcome of patients with HIV infection and tuberculosis caused by multipledrug-resistant bacilli. Ann Intern Med 1992;117:184-90.

10. Telzak EE, Turett G, Torian L, Fazal B, Weisfuse I. Multidrug-resistant tuberculosis (MDRTB) in the South Bronx, New York City: clinical characteristics and improved outcome. Presented at the Eighth International Symposium on Infections in the Immunocompromised Host, Davos, Switzerland, June 19-22, 1994. abstract.

11. Edlin BR, Attoe LS, Grieco MH, Williams J, Schneider N, Gilligan M. Recognition and treatment of primary multidrug-resistant tuberculosis (MDRTB) in HIV-infected patients. In: Abstracts of the Ninth International Conference on AIDS/Fourth STD World Congress, Berlin, Germany, June 6-11, 1993. London: Wellcome Foundation, 1993. abstract.

12. Sharp V, Sepkowitz KA, Lockhart B. Survival with MDR-TB and AIDS depends on proper selection of initial therapy. Presented at the Eighth International Symposium on Infections in the Immunocompromised Host, Davos, Switzerland, June 19-22, 1994. abstract.

13. Goble M, Iseman MD, Madsen LA, Waite D, Ackerson L, Horsburgh CR Jr. Treatment of 171 patients with pulmonary tuberculosis resistant to isoniazid and rifampin. N Engl J Med 1993;328:527-32.

14. Heifets LB, Good RC. Current laboratory methods for the diagnosis of tuberculosis. In: Bloom BR, ed. Tuberculosis: pathogenesis, protection and control. Washington, D.C.: ASM Press, 1994:85-110.

15. Brudney K, Dobkin J. Resurgent tuberculosis in New York City: human immunodeficiency virus, homelessness and the decline of tuberculosis control programs. Am Rev Respir Dis 1991;144:745-9.

16. Alland D, Kalkut GE, Moss AR, et al. Transmission of tuberculosis in New York City: an analysis by DNA fingerprinting and conventional epidemiologic methods. N Engl J Med 1994;330:1710-6.

17. Small PM, Hopewell PC, Singh SP, et al. The epidemiology of tuberculosis in San Francisco: a population-based study using conventional and molecular methods. N Engl J Med 1994;330:1703-9.

18. Shafer RW, Small PM, Larkin C, et al. Temporal trends and transmission patterns during the emergence of multidrug-resistant tuberculosis in New York City: a molecular epidemiologic assessment. J Infect Dis 1995;171: 170-6.

\section{IMAGES IN GLINICAL MEDICINE}

Images in Clinical Medicine, a weekly Journal feature, presents clinically important visual images, emphasizing those a doctor might encounter in an average day at the office, the emergency department, or the hospital. If you have an original unpublished, high-quality color or black-and-white photograph representing such a typical image that you would like considered for publication, send it with a descriptive legend to Kim Eagle, M.D., University of Michigan Medical Center, Division of Cardiology, 3910 Taubman Center, Box 0366, 1500 East Medical Center Drive, Ann Arbor, MI 48109. For details about the size and labeling of the photographs, the requirements for the legend, and authorship, please contact Dr. Eagle at 313-936-5275 (phone) or 313-936-5256 (fax), or the New England Journal of Medicine at images@edit.nejm.org (e-mail). 